\title{
USE OF PHYSIOTHERAPY TO IMPROVE FATIQUE IN PATIENTS WITH MULTIPLE SCLEROSIS
}

\author{
Denka Marinova \\ National Sports Academy "Vassil Levski", Sofia, Bulgaria
}

\begin{abstract}
Introduction: Multiple sclerosis (MS) is a serious disease of the brain and the spinal cord whose results in almost all patients is fatigue. In MS, fatigue is a frequent disorder which can affect the quality of life and increase disability. Objectives: To investigate if individually tailored physiotherapy and exercise alleviates the condition of fatigue in MS patients. Methodology: 28 subjects' whit secondary progressive MS with stabilized condition received individual physiotherapy. The Expanded Disability Status Scale (EDSS) and Fatigue Severity Scale (FSS) were registered at baseline and immediately after treatment. Patients are given a specific therapeutic program: one hour a day, three times a week. Results: Mean FSS at baseline was 4.77 ( \pm SD1.45) and improved during treatment to $4.43( \pm S D 1.24)$ $(p<0.001)$. Mean EDSS at baseline was $5.16( \pm S D 0.74)$ and improving treatment to $4.90( \pm S D 0.86)(p<0.001)$ in the sixth week. Although the tendency to worsen fatigue was great, an improvement in the condition was observed. EDSS was significantly reduced compared to baseline $4.82( \pm$ SD0.93) $(p<0.001)$. Discussion and conclusions: Fatigue and neurological deficit improved as a result of individually tailored physiotherapy in MS. The conducted study and the results indicate that goal-oriented physiotherapy, positively influences fatigue patients with multiple sclerosis.
\end{abstract}

Key words: multiple sclerosis, fatigue, physiotherapy, exercise

\section{Introduction}

Multiple sclerosis (MS) is a chronic, inflammatory, demyelinating disease of the central nervous system. It causes various combinations of symptoms which can be manifested as damage to the myelin of the white brain substance, the ocular nerves, the pyramid paths, the sensory conductors, the cerebrum and the spinal cord. (Milanov, 2005). In recent years, the interest in MS has increased significantly. This is due to the great social importance of the disease, the progress in the development of computer tomography, as well as the introduction of medications that modify the development of the disease. Fatigue is one of the most frequent symptoms of MS, occurring in about $80 \%$ of the affected people, and leading to grave disability (Branas, 2000). A number of research works show that fatigue is a serious problem that is the reason for the functional inability of patients, the authors including it among the three most important symptoms of the disease (Krupp, 2003; Fiske, 1994). Fatigue is actually general, intense, and continual weakness manifested after physical and intellectual activity. It is defined as resistant to all influence, and the feeling of loss of vitality and energy. This objective loss of physical and psychic energy hampers the carrying out of everyday routine activities and worsens the quality of life of patients. Physiotherapy in MS refers to physical activity that is planned, structured, and carried out over a long period of time, with a view to improving and maintaining physical status. (Carspersen, 1985). It has been documented that regular aerobic exercises decrease health risks in MS patients and positively affect the feeling of fatigue (Paffenbarger, 1986). The main strategy of treating MS is to stop the development of the disease and to control the concomitant symptoms, part of which is fatigue. This can be achieved by prevention of exacerbations and slowing down the process of the disease. (Daskalova, 2009). The study of literary sources on the issue testify that at present the treatment of fatigue in MS remains one of the most urgent and complex problems in modern medical science. Their analysis shows that physiotherapy still remains insufficiently investigated as compared with medicinal treatment.

\section{Methodology and methods \\ Participants}

The research has been conducted among MS patients over a three-year period. Subjects of the research were 28 diseased people with clinically ascertained MS diagnosis of relapsing-remitting kind. The age of the patients ranges from 24 to 55 , the period of the disease being from 2 to 20 years. All subjects meet the following criteria in order to be 
included in the research: ascertained MS diagnosis; years of age between 20 and 60; remission phase; the ability to move on their own; lack of heavy cardiovascular complaints; and stable hemodynamic indicators. The research does not include patients with contra-indications for physical therapy, concomitant cardiovascular diseases, high blood pressure, damaged general condition, or advanced stage of MS. Patients with deep depressions or psychic problems were also excluded. The approved patients have been observed by a specialist in neurology and have taken prescribed medications. Each subject has submitted an informed consent for participation in the research in the written form.

\section{Study design}

This research aims to prove that fatigue in MS patients favorably affected by specialized motor activity, in conformity with individual capacities, regardless of the disability degree. The patients were assessed at the beginning and the end of the test according to the Expanded Disabiliy Status Scale (EDSS) and the FSS (Fatigue Severity Scale).

\section{Methodology}

The physiotherapeutic program is based on conducted research, the current condition of patients, and is personally adapted to each subject. There have been carried out 10 physiotherapeutic procedures, three times a week, in outpatients' wards or at home. The duration of each procedure is 50 to 60 $\mathrm{min}$. The pressure during exercises is controlled according to the subjective fatigue indicators, as well as indicators concerning the pulse rate and the arterial blood pressure, at the beginning and the end of each procedure. The purpose of PT is to reduce fatigue and to achieve maximal functional independence through stabilizing patients' condition. The procedures consist of standardized aerobic training program. The program includes dynamic exercises for the large muscular groups, exercises on the PNF methodology, veloergometer pedaling, and breathing exercises. The subjects make up to 30 -minutes therapeutic exercises, 15 to $20 \mathrm{~min}$. velo's pedaling, and breathing exercises. The initial intensity of the training course on the exercise bike is fixed on $\mathrm{VO} 2$ max, from $50 \%$ to $70 \%$ consumption of oxygen. In case of pains in the heart area, constriction, fatigue, deviations in the blood pressure, or some other signs of overloading, the procedure is cancelled.

\section{Results}

While statistically processing the results, we have used the following indicators: minimal value (Min), maximal value (Max), range $(R)$, average arithmetic value $(\mathrm{X})$, standard error of the average arithmetic value $(\mathrm{Mx})$, standard deviation (SD), co-efficient of variation (V\%), asymmetry (As), excess (Ex), p $\leq 0.05$ referring to the level of significance. For the check of statistical hypotheses, the criterion of Student $t$ - for dependent and independent samples, and $\mathrm{X}$ - Pearson criterion for comparison of frequency distributions at non-parametric indicators for group.

The changes having taken place as a result of the conducted physiotherapy to MS patients are laid down in Table 1-2 and Diagram 1-2.

Table 1. Changes in the average values and standard deviations of fatigue level measured at the beginning and the end of treatment

\begin{tabular}{|l|l|l|l|l|l|l|l|l|l|}
\hline & $\mathbf{M i n}$ & $\mathbf{M a x}$ & $\mathbf{R}$ & $\mathbf{X}$ & $\mathbf{m x}$ & $\mathbf{S D}$ & $\mathbf{V} \%$ & $\mathbf{A s}$ & $\mathbf{E x}$ \\
\hline Начало & 2.5 & 7 & 4.5 & 4.77 & 0.29 & 1.45 & 29.43 & -0.42 & 0.85 \\
\hline Край & 0 & 4 & 4 & 4.43 & 0.14 & 1.24 & 19.89 & -0.21 & 0.06 \\
\hline
\end{tabular}

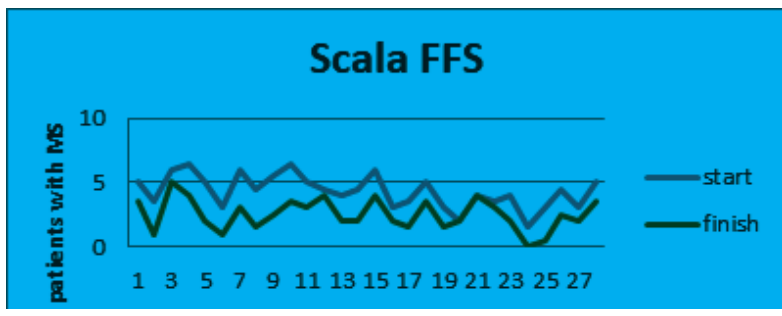

Diagram 1. Dynamics of fatigue at the beginning and the end of treatment

Table 2. Changes in the average values and standard deviations in the degree of neurological deficit according to the scale of Kurtzke (EDSS), measured at the beginning and the end of treatment

\begin{tabular}{|l|l|l|l|l|l|l|l|l|l|}
\hline & $\mathbf{M i n}$ & $\mathbf{M a x}$ & $\mathbf{R}$ & $\mathbf{X}$ & $\mathbf{m x}$ & $\mathbf{S D}$ & $\mathbf{V} \%$ & $\mathbf{A s}$ & $\mathbf{E x}$ \\
\hline Начало & 3.5 & 6.6 & 4.5 & 5.16 & 0.29 & 0.74 & 35.89 & 0.25 & -1.33 \\
\hline Край & 1 & 5 & 3.1 & 4.82 & 0.33 & 0.86 & 45 & 0.23 & -1.25 \\
\hline
\end{tabular}

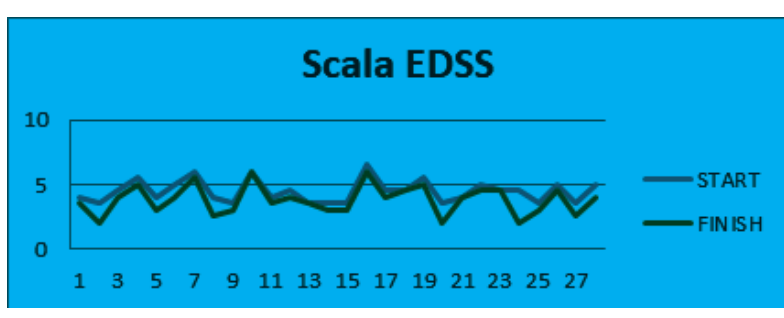

Diagram 2. Dynamics of neurological deficit at the beginning and the end of treatment 


\section{DISCUSSION}

Chronic fatigue is one of the most important symptoms in the condition of MS patients, and it is hard to cure (Milanov, 2005). The decrease of the feeling of fatigue is a major criterion of the physiotherapy efficiency. Regular motor activity can well be an alternative strategy for the treatment of fatigue in MS patients (Stroud, Minahau, 2009). Table 1 clearly shows that the co-efficient of variation is high, which can be explained by the small difference in the absolute values of this value. The co-efficient of As and Ex show, however, that there is a great concentration of values, i.e. the distribution of results is symmetrical and concentrated. The follow-up of the fatigue dynamics shows the existence of heavy fatigue at the beginning of the therapy, with a tendency to decrease after starting the treatment (chart 1). On comparing the results at the beginning and the end of the research, statistically credible difference in the average values of results is identified. This makes the initial results comparable and the differences objective. At maximal fatigue degree equal to 7 , the average value is $4.77( \pm \mathrm{SD} 1.45)$, and at the end of the therapy it is $4.43( \pm \mathrm{SD} 1.24)$ $(\mathrm{p}<0.001)$. At the end of the treatment course, as a result of the ministered therapy, statistically significant decrease in the patients' fatigue is ascertained. The average value reached $1.20 \leq 1.87$. The significant change to fatigue decrease can be explained by the favorable influence of aerobic physiotherapy Ponichtera-Mulcare (1993). The successful research of Petajan, (1996), along with the earlier works of Ponichtera-Mulcare, Petajan (1993), set the standard trends in the treatment of fatigue in MS patients.

The analysis of the neurological deficit results identifies a large difference in evaluations, the degree of neurological deficit ranging between 3.5 and 6.5, at the maximum degree of 10 . The average value at the beginning is $5.16( \pm \mathrm{SD} 0.74)$, while at the end of the therapy, it is $4.90( \pm$ SD0.86) $(\mathrm{p}<0.001)$. On comparing results, it is ascertained that at the beginning of the therapy, patients have graver functional deficit, while at the end of the final course, a decrease in this deficit is identified. On the course of treatment, a better motor function is observed, which is ascribed to the fatigue decrease and the improvement of the functional condition. A treatment of MS patients is considered successful when the development of the disease slows down or even stops completely. This shows that the patients enter a stable condition. Having in mind the progressing nature of the disease and the general tendency to aggravation of the patients' conditions, this result is optimistic in relation to the therapeutic effect of physiotherapy suggested by us. Regular physical exercises can not only prevent worsening disability but also act as prophylaxis to the development of complications (Mackereth, 2009). This is achieved through the improvement of both physical (Liu, C., 2003) and mental health.

\section{Conclusion}

So far, there are no data for a therapeutic method resulting in the successful cure of MS patients. Efforts are directed to the treatment of symptoms, prevention of bouts, and annihilation of the disease development. The obtained results are auspicious, though they give no ground for final conclusions because of the small number of tested patients and the comparatively short course of therapy. The ascertained favorable effect of the applied physiotherapeutic methods is related to the decrease in fatigue and stabilizing the general condition of MS patients.

\section{References}

Branas, P., Jordan, R., Fry-Smith A., Hyde C. (2000), Treatments for fatigue in multiple sclerosis: a rapid and systematic review. Journal of Health Technol Assess, Vol. 25 No.6, pp.41-61.

Carspersen, C.J., Powell K, E., Christenson, G.M. (1985), Physical activity, exercise and physical fitness: definitions and distinctions for health-related research. Public Health Journal, Vol. 48 Suppl., pp, 126-131.

Daskalova, V., Aleksandrov, A., Kostadinova, C. (2009), Multiple sclerosis, Median PRESS, pp. 296-348.

Fiske, J.D., Ritro, P.G. et al. (1994), The impact of fatigue on patient with multiple sclerosis, Journal of Neurol. Science, Vol. 21 No.1, pp. 9-14.

Krupp, L.B. (2003), Fatique in Multiple sclerosis: definition, pathophysiology and treatment. CNC Drugs Journal, Vol. 17, pp. 225-34.

Liu, C., Playford, E.D., Thompson, A.J. (2003). Does neurorehabilitation have a role in relapsing remitting multiple sclerosis? Journal of Neurology, Vol.25, pp.214-218. Mackerethy, P.A., Boothy, K., Hilliery, V.F., Caress, A.L. (2009). Reflexology and progressive muscle relaxation training for people with multiple sclerosis: a crossover trial, Complement Ther. Clin. Pract, Vol.15, pp.14-21.

Milanov, I. (2005), Multiple sclerosis, Medicine and Physical Education, 2005, 28-30.

O'Connell, R., Murphy, R.M., Hutchinson, $M$ et al. (2003), A controlled study to assess the effects of aerobic training on patients with multiple sclerosis. Paper presented at: 14th International World Confederation for Physical Therapy; June Barcelona, Spain, pp. 7-12. 
Paffenbarger, R.S., Hyde, R.T., Wing, A.L. Hsieh, C.C. neurotrophic factors, quality of life and coordinative (1986), Physical activity, all-cause mortality, and longev- function in multiple sclerosis. J Neurol Sci. Suppl. 225, ity of college alumni. New England. Journal of Medicine. Vol. 14 No.10, pp. 605-613. pp.211-218. Petajan, J.H., Gappmaier, E., White, A.T. et al. (1996), lar physical activity on fatigue, depression and quality of Impact of aerobic training on fitness and quality of life life in person with multiple sclerosis. Health Qual. Life, in multiple sclerosis. Journal of Neurology. Vol. 39, pp. Outcome. pp. 57-68. 432-441.

Ponichtera-Mulcare, J.A., Glaser, R.M.. et al. (1993), Maximal aerobic exercise in persons with multiple sclerosis. Journal of Kinesiology, Vol. 45, pp. 12-21.

\section{Correspondence:}

Denka Marinova

National Sports Academy "Vassil Levski",

Schulz, K.H., Gold, S.M., Witte, J, et al. (2004), Impact Sofia, Bulgaria

of aerobic training on immune-endocrine parameters, E-mail: denka_marinova@abv.bg 